structures of the type predicted in some theories ${ }^{4}$.

The ability to manipulate individual vortices might also lead to the realization of new types of flux-line-based devices, called fluxtronics. There have been proposals for devices using vortices as the elementary units in classical logic devices ${ }^{5}$, as well as proposals to manipulate individual spins or charges by coupling the vortex to these objects and then manipulating the vortex line ${ }^{6}$. Similar techniques for moving individual line-like objects could be used in other systems, such as dragging or shaking individual domain walls in magnets, or moving dislocation lines in materials to create specific patterns that will enhance the mechanical properties of the material.
Individual particle manipulation has been widely developed as a tool in systems with larger-scale objects, such as in biological matter where DNA strands are mechanically manipulated ${ }^{7}$, or in soft-matter systems where grabbing and shaking a single colloid can be used as a microrheological probe ${ }^{8}$. The work of Auslaender et al. ${ }^{1}$ points to the feasibility of using many of these ideas on a much smaller scale, not only for vortex systems, but also for a wider class of solid states. One can imagine using multiple probe tips to create a nanorheological probe for vortex or quantum glasses. These local probe techniques may usher in a new era where the individual manipulation of quantum objects could be used to explore local and non-local responses, quantum entanglement, many-body effects and the role of heterogeneities in determining the sample behaviour.

Charles Reichhardt is in the Los Alamos National Laboratory, T-13, MS B213, Los Alamos,

New Mexico 87545, USA.

e-mail:charlesr@cnls.lanl.gov

\section{References}

1. Auslaender, O. M. et al. Nature Phys. 5, 35-39 (2009).

2. Kafri, Y., Nelson, D. R. \& Polkovnikov, A. Phys. Rev. B 76, 144501 (2007)

3. Olson Reichhardt, C. J. \& Hastings, M. B. Phys. Rev. Lett. 92, 157002 (2004)

4. Kivelson, S. A. et al. Rev. Mod. Phys. 75, 1201-1241 (2003).

5. Hastings, M. B., Olson Reichhardt, C. J. \& Reichhardt, C. Phys. Rev. Lett. 90, 247004 (2003).

6. Berciu, M., Rappoport, T. G. \& Janko, B. Nature 435, 71-75 (2005)

7. Bustamante, C., Bryant, Z. \& Smither, S. B. Nature 421, 423-427 (2003).

8. Levine, A. J. \& Lubensky, T. C. Phys. Rev. Lett. 85, 1774-1777 (2000).

\title{
MATTER WAVES
}

\section{Cloaking matters}

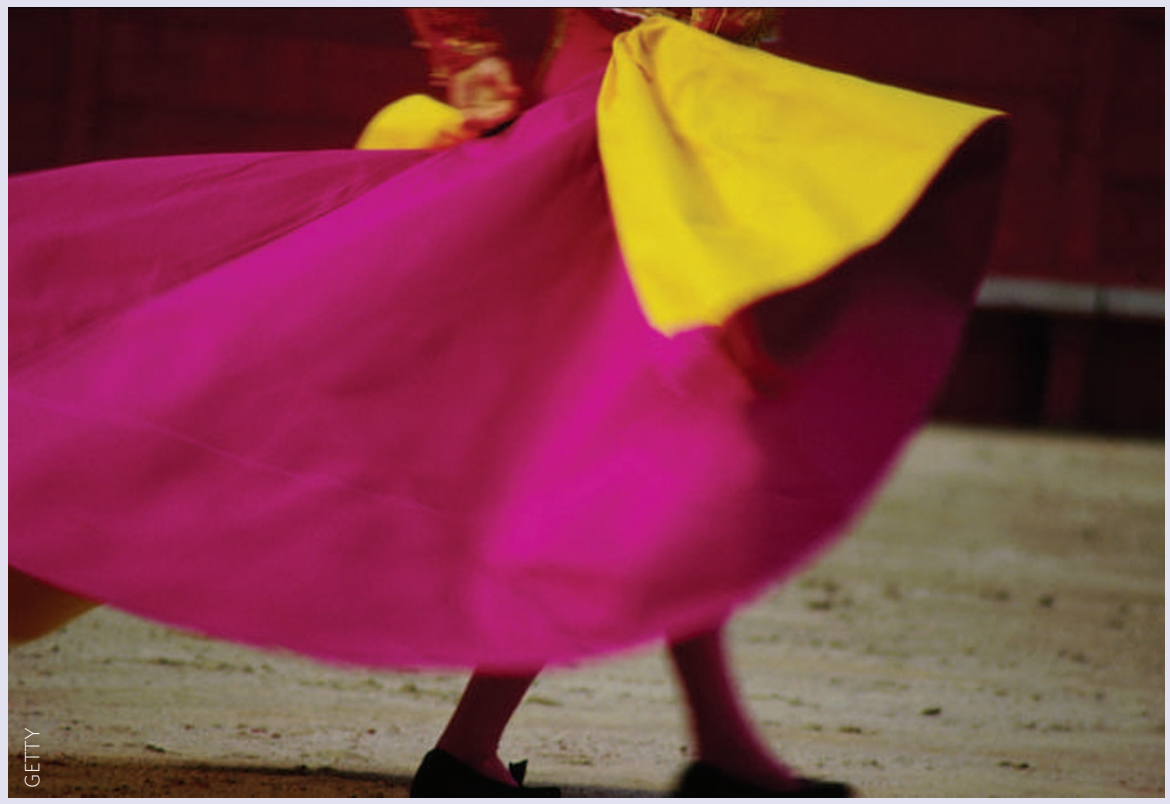

The idea of an invisibility cloak - a device capable of bending light, keeping whatever is inside safe from prying eyes - is one that was always destined to capture the public imagination. Over the past few years it has developed from sci-fi mainstay to experimental fact (albeit only under certain conditions), and the excitement in the popular press has just kept growing

As quantum mechanics blurs the boundaries between the properties of particles and those of waves, such as light, it is not outrageous to wonder - as Allan Greenleaf and colleagues do (Phys. Rev. Lett. 101, 220404; 2008) whether these same cloaking ideas can be applied to matter.

How does a particle react to a potential barrier of a certain size and shape? It is a standard question posed to undergraduate students when introducing Schrödinger's equation. But Greenleaf et al. have turned this problem on its head: what type of barrier will act on a particle with a specific energy to recreate the cloaking effect. Thinking in two dimensions, they propose a series of rings, each with a different potential, that together mask whatever is within from a probing matter wave.

The idea of a cloak for matter waves is not new, however, construction via the approach published previously Shuang Zhang and colleagues (Phys. Rev. Lett. 100,$123002 ; 2008$ ) is likely to be difficult in practice. Greenleaf et al. hope that their concept will offer an easier path.

Its simplicity comes at a price - the cloaking is not perfect - but even this drawback has its advantage: particles at an energy at which the device doesn't work can be trapped within the cloak. Such traps have already proved a boon for fundamental research on atomic physics, and a novel approach is always likely to come in handy.

The matter cloak may be a long way from fruition, but it is a further example of how optical phenomena can be translated to matter waves. The idea complements proposals to use graphene as a Veselago's lens for electron beams and 'extraordinary' transmission of rubidium atoms through an array of sub-de Brogliewavelength slits.

DAVID GEVAUX 